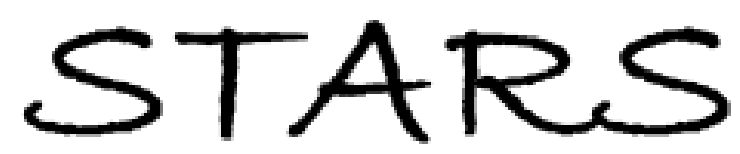

University of Central Florida

STARS

Faculty Bibliography 2000s

Faculty Bibliography

$1-1-2005$

\title{
Variable-focus liquid lens by changing aperture
}

Hongwen Ren

University of Central Florida

Shin-Tson Wu

University of Central Florida

Find similar works at: https://stars.library.ucf.edu/facultybib2000 University of Central Florida Libraries http://library.ucf.edu

This Article is brought to you for free and open access by the Faculty Bibliography at STARS. It has been accepted for inclusion in Faculty Bibliography 2000 s by an authorized administrator of STARS. For more information, please contactSTARS@ucf.edu.

\section{Recommended Citation}

Ren, Hongwen and Wu, Shin-Tson, "Variable-focus liquid lens by changing aperture" (2005). Faculty Bibliography 2000s. 5582.

https://stars.library.ucf.edu/facultybib2000/5582

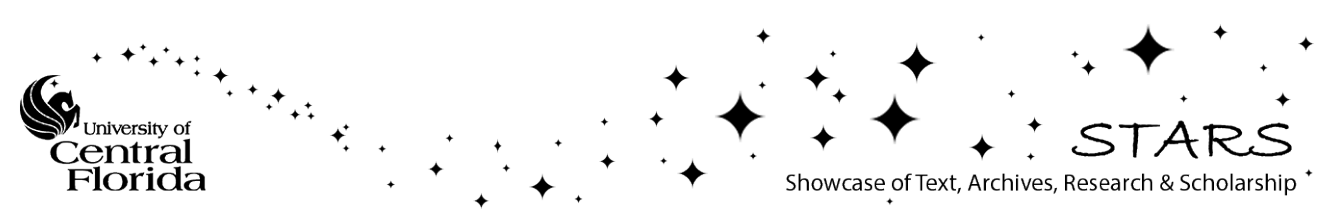




\title{
Variable-focus liquid lens by changing aperture
}

\author{
Hongwen Ren and Shin-Tson Wu ${ }^{\mathrm{a})}$ \\ College of Optics and Photonics, University of Central Florida, Orlando, Florida 32816
}

(Received 21 February 2005; accepted 13 April 2005; published online 17 May 2005)

\begin{abstract}
We propose an adaptive liquid-filled lens, which consists of an elastic membrane, a solid plate, and an annular sealing ring; a liquid with a fixed volume stored in lens chamber. The key part is the annular sealing ring which looks like an iris diaphragm. The surfaces of annular sealing ring are sealed with an elastic membrane. The radius of the annular sealing ring is changeable. By tuning the radius of the annular sealing ring, the stored liquid in the lens will be redistributed, thus changing the curvature of the elastic membrane. Therefore, the lens cell causes light to converge or diverge. A liquid lens with a positive variable focus was demonstrated, this kind of lens has the advantages of simple fabrication process, compact structure, easy operation, and low cost. (C) 2005 American Institute of Physics. [DOI: 10.1063/1.1935749]
\end{abstract}

Since liquid was proposed for making a variable focus lens, ${ }^{1}$ liquid-based zoom lenses have been studied extensively for eye glasses, cameras, camcorders, projectors, as well as other machine vision. Compared with other adaptive lenses, such as liquid-crystal lens ${ }^{2,3}$ and polyelectrolyte gel lens, ${ }^{4}$ the variable-focus liquid lens present the simplest and the highest-quality performances. According to the operating mechanisms and lens structure, liquid lenses can be classified into three types. The first type is the liquid lens having a fixed volume of liquid sandwiched between a planar substrate and a thin elastic membrane. ${ }^{5}$ By moving the periphery of the elastic membrane, the liquid inside the lens assembly is redistributed such that the curvature of the film is changed, thus causing light to converge or diverge. A drawback of this kind of lens is that one cannot easily keep the periphery of the elastic membrane parallel to that of the planar substrate during the distance change. Moreover, the operating system for tuning the focus is complicated. Liquid lenses operated by pumping liquid in and out of the lens chamber to change the curvature of the elastic membrane surface belong to the second type. ${ }^{6-9}$ This type of fluidic lens exhibits a high lens power without changing lens aperture. Both single large aperture lens and microlens arrays can be fabricated easily. However, a fluidic pumping system is usually needed, thus, the lens system is sensitive to vibration. As for the third type, the focal length of the liquid lens is tuned by applying an external voltage. ${ }^{10-12}$ This type of lens has fast response time and continuous focus change. However, making a large aperture lens is a challenging task.

In this letter, we demonstrate a fourth type of variablefocus liquid lens. Unlike other liquid lenses, we vary the focus of the liquid lens by changing its aperture size. In our liquid lens, the key element is the circular periphery seal which is similar to an iris diaphragm with rotatable impellers. A lever actuator is used to control the movement of the rotatable impellers and impart the pressure to the fluid-filled lens. Thus, the redistributed liquid will change the shape of the lens curvature, and then change the focal length of the lens. Compared with other liquid lenses, our liquid-filled lens has several advantages, such as simple fabrication process, compact structure, easy operation, and low cost. Based on

\footnotetext{
${ }^{a)}$ Electronic mail: swu@ mail.ucf.edu
}

this simple lens structure, different types of liquid lenses can be fabricated. ${ }^{13}$

Figure 1(a) depicts the schematic diagram of the liquid lens which consists of a glass plate, a circular periphery seal, and a clear elastic membrane. The circular periphery seal is wrapped using an elastic membrane to confine the liquid. The key element of the liquid lens is the circular periphery seal which looks like a conventional iris diaphragm, as shown in Fig. 2. The rotatable impellers are the incorporated parts of the circular periphery seal. A lever actuator is used to control the movement of the rotatable impellers. In Fig. 2(a), the radius of the aperture is $r_{0}$. If the elastic membrane is flat, as shown in Fig. 1(a), there is no focusing effect. When the radius of the aperture is reduced to $r_{1}\left(r_{1}<r_{0}\right)$ by rotating the lever actuator in the clockwise direction, as shown in Fig. 2(b), the surface of the elastic membrane becomes convex. Thus, a positive (or converging) lens is formed, as shown in Fig. 1(b). This is because the rotated impellers suppress the filled liquid which, in turn, causes the elastic membrane to swell outward. On the other hand, if the radius of the aperture is enlarged to $r_{2}\left(r_{2}>r_{0}\right)$ by rotating the lever actuator in the counterclockwise direction, as shown in Fig. 2(c), then the membrane curvature becomes concave so that a negative (or diverging) lens is formed.

To derive the relationship between the radius of the liquid lens $r$ and the radius of the lens curvature $R$, we simply
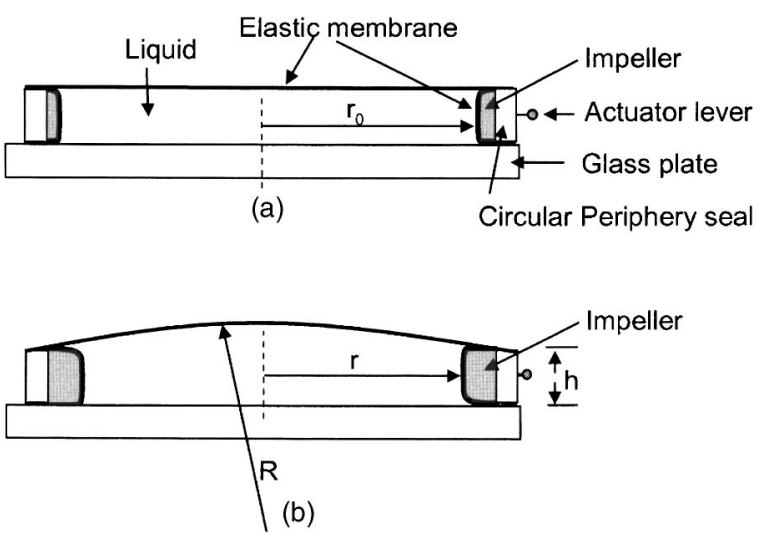

FIG. 1. The cross-sectional view of the liquid lens cell with (a) no focus effect with an aperture radius $r_{0}$, (b) focus effect with an aperture radius $r$ $<r_{0}$. 


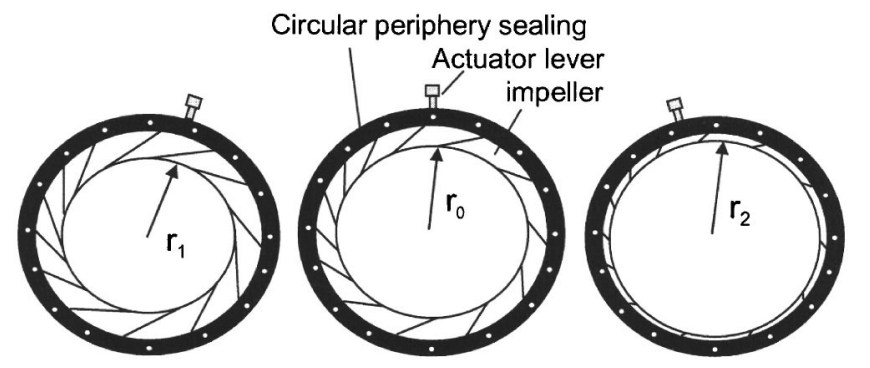

FIG. 2. The top view of the circular periphery seal similar to an iris diaphragm structure. The radius of the aperture is (a) $r_{0}$, (b) $r_{1}\left(r_{1}<r_{0}\right)$, and (c) $r_{2}\left(r_{2}>r_{0}\right)$.

use the lens structure shown in Fig. 1 in which the lens parameters are defined. Considering that the volume of the liquid is not constringent and the glass plate is strictly rigid, when the radius of the aperture is changed from $r_{0}$ to $r$; the volume of the redistributed liquid can be expressed as

$$
\pi\left(r_{0}^{2}-r^{2}\right) h=\frac{\pi}{3}\left(R-\sqrt{R^{2}-r_{0}^{2}}\right)^{2}\left(3 R-\left(R-\sqrt{R^{2}-r_{0}^{2}}\right)\right) .
$$

In Eq. (1), $h$ is the effective thickness of the liquid lens. Using Mathematica software, we can derive the relationship between $R$ and $r$, but the formula is too complicated to show here. Without losing generality, we illustrate our design concept using a numerical example. Let us suppose the maximum radius of the aperture $r_{0}=12.5 \mathrm{~mm}$ and the thickness of the liquid lens $h=5.5 \mathrm{~mm}$. The relationship between $R$ and $r$ is plotted in Fig. 3. When the radius of the lens is decreased from 12.5 to $9.5 \mathrm{~mm}$, the radius of curvature $R$ is decreased sharply. As $r<9.5 \mathrm{~mm}, R$ decreases at a much slower rate.

Because of the liquid redistribution, the elastic membrane curvature is altered. The corresponding liquid lens has the following focal length:

$$
f=\frac{R}{n-1}
$$

where $n$ is the refractive index of the liquid. From Eqs. (1) and (2), for a fixed $r_{0}$, the focal length of the lens can be reduced by decreasing $r$. Choosing a high refractive index liquid or increasing the lens thickness can also enhance the lens power. For demonstration purposes, we choose a liquid (ethanol) with $n=1.36$ as an example. Figure 3 plots the simulated focal length (right-hand side ordinate) and the radius of the lens aperture (left-hand side ordinate). From Fig.

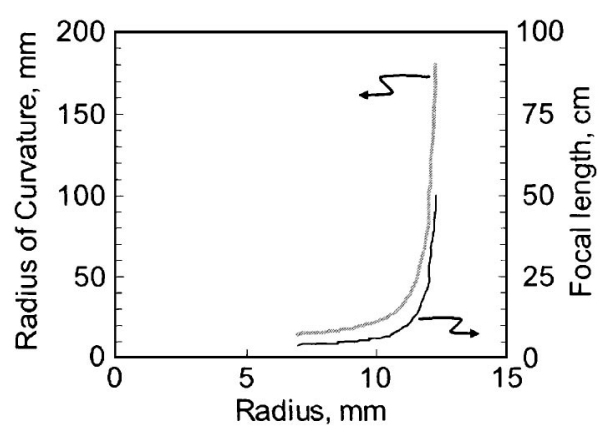

FIG. 3. The simulated radius of the curvature $(R)$ and focal length $(f)$ as a function of the lens aperture size. The thickness of the rotatable impellers is $5.5 \mathrm{~mm}, h=5.5 \mathrm{~mm}, r_{0}=12.5 \mathrm{~mm}$, and $n=1.36$.
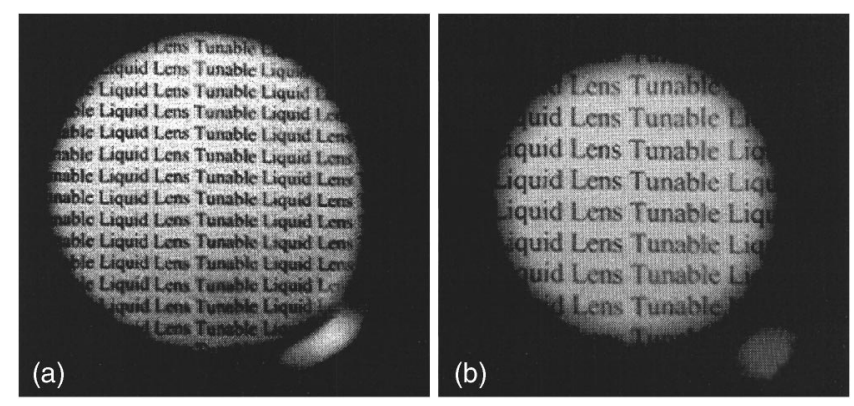

FIG. 4. Imaging behavior of the liquid lens when the lever actuator is (a) at the neutral position $\left(r_{0}=12.5 \mathrm{~mm}\right)$ and $(\mathrm{b})$ rotated to shrink the aperture of the lens. The employed liquid is ethanol.

3, the focal length and the radius of curvature have a similar trend.

We fabricated a liquid lens according to the structure shown in Fig. 1(a). An iris diaphragm (from Edmund Industrial Optics) was used as the circular periphery seal, the maximum aperture and the thickness of the iris diaphragm is $25 \mathrm{~mm}$ and $5.5 \mathrm{~mm}$, respectively. A clear distensible balloon is used as the elastic membrane. The iris diaphragm was wrapped using the elastic membrane. Ethanol as the liquid $(n=1.36)$ was stored into the lens cell chamber. The lens chamber was fastened by screws. In the original state, the surface of the elastic membrane of the lens was flat.

To evaluate the image quality of the liquid lens, we typed a few lines of "tunable liquid lens" on a piece of cardboard as an object, and the lens was set in front of the object. To test the gravity effect of liquid on the membrane curvature, we intentionally set the lens in vertical direction. A digital camera sits right behind the sample. Two photos were taken with the lever actuator at two different positions, as shown in Figs. 4(a) and 4(b), respectively. In Fig. 4(a), the lever actuator is at the neutral position, so that the cell is flat and has no lens effect. A clear image of the typed words is observed and the size of the image is the same as that of the object. As the lever actuator was rotated and the aperture of the lens became smaller, the observed image is magnified, as shown in Fig. 4(b). By continuously rotating the lever actuator, the aperture of the lens can become much smaller and the observed image can be magnified significantly. The enlarged image means the lens power is increased and the focal length is decreased. The liquid lens in this case belongs to convex lens.

Different from the block rotatable impellers of the lens structure shown in Fig. 1, in our demonstration, we used a thin $(\sim 0.5 \mathrm{~mm})$ impeller. Compared with Eq. (1), when the impellers are screwed inward, the lens aperture shrinks. As a result, the volume of the redistributed liquid is halved as compared to the block impellers, and the relationship between the radius of the curvature $R$ and the radius of the lens aperture $r$ can be written as:

$$
\frac{\pi}{2}\left(r_{0}^{2}-r^{2}\right) h=\frac{\pi}{3}\left(R-\sqrt{R^{2}-r_{0}^{2}}\right)^{2}\left(3 R-\left(R-\sqrt{R^{2}-r_{0}^{2}}\right)\right) .
$$

For a given $r_{0}$ and $h$, we can derive the relationship between $r$ and $R$ from Eq. (3). Once the $R$ is obtained, the lens focal lens can be calculated from Eq. (2). Figure 5 plots the simulated and experimental results of the liquid lens. They agree quite well. Compared with the focal length plotted in Fig. 3, 


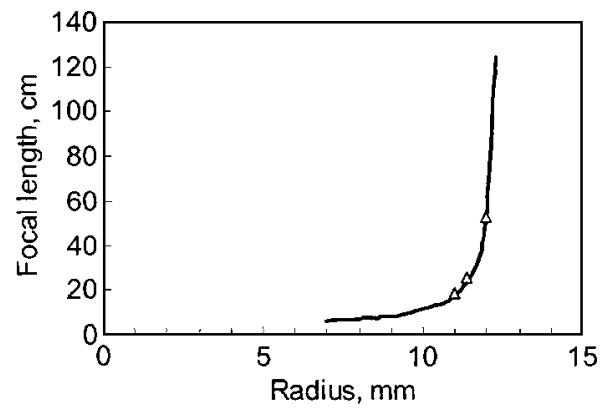

FIG. 5. Relationship between the focal length and the radius of the lens aperture with a thin $(\sim 0.5 \mathrm{~mm})$ rotatable impeller. The triangles and solid line represent the experimental and theoretical results, respectively. The thickness of the lens $h=5.5 \mathrm{~mm}$, the maximum radius of the aperture $r_{0}$ $=12.5 \mathrm{~mm}$, and the refractive index of the liquid $n=1.36$.

the focal length of our demo liquid lens is halved if the changed radius of the lens aperture is the same. This is because a thin impeller was employed. To obtain a liquid lens with a wide tunable focal length, the thickness of the rotatable impellers should be the same as the circular periphery seal.

In comparison with other variable liquid lens technologies, the major advantages of our lens are in the simple fabrication process, simple lens structure, easy operation, and thus the relatively low cost. In addition, the lens has a very wide range of variable focal lengths. By rotating the impellers of the liquid lens in or out, we can get not only a convex lens but also a concave lens easily. The actuator lever of the liquid lens can be rotated mechanically or electrically, i.e., the focus change can be operated manually or electromechanically. Moreover, if the rotatable impellers are made of clear glass, plastics, or polymer, then the apparent aperture will not be noticeable even when the impellers are rotated. In our experiment, the range of the variable focal length can be significantly widened if we use a higher index liquid, increase the effective thickness of the liquid lens, or change the radius of the lens aperture to a larger degree.

In summary, we have demonstrated a variable-focus liquid lens by changing its aperture size. Both positive and negative foci can be realized by controlling the size of the lens aperture. For proving principles, we fabricated a simple liquid lens. By changing its aperture size, a positive lens was obtained. This kind of lens is easy to fabricate, lightweight, and has very weak color dispersion. Its applications for camera zoom lens and tunable-focus eyeglasses are foreseeable.

The authors would like to think Yi-Hsin Lin, Yung-Hsing Fan, and Ying Zhou for their technical assistance and useful discussions.

${ }^{1}$ B. M. Wright, English Patent No. 1,209,234 (11, March 1968).

${ }^{2}$ S. Sato, Jpn. J. Appl. Phys. 18, 1679 (1979).

${ }^{3}$ H. Ren, Y. H. Fan, S. Ganza, and S. T. Wu, Appl. Phys. Lett. 84, 4789 (2004).

${ }^{4}$ Y. Kawai, S. Morita, and S. Hattori, J. Eye 5, 1021 (1988).

${ }^{5}$ S. Kurtin, U.S. Patent 5, 138, 494 (11, August 1992).

${ }^{6}$ N. Sugiura and S. Morita, Appl. Opt. 32, 4181 (1993).

${ }^{7}$ D. Y. Zhang, V. Lien, Y. Berdichevsky, J. Choi, and Y. H. Lo, Appl. Phys. Lett. 82, 3171 (2003).

${ }^{8}$ K.-H. Jeong, G. L. Liu, N. Chronis, and L. P. Lee, Opt. Express 12, 2494 (2004).

${ }^{9}$ J. Chen, W. Wang, J. Fang, and K. Varahramyan, J. Micromech. Microeng. 14, 675 (2004).

${ }^{10}$ M. Vallet and B. Berge, Polymer 37, 2465 (1996).

${ }^{11}$ T. Krupenkin, S. Yang, and P. Mach, Appl. Phys. Lett. 82, 316 (2003).

${ }^{12}$ S. Kuiper and B. H. W. Hendriks, Appl. Phys. Lett. 85, 1128 (2004).

${ }^{13}$ H. Ren, and S. T. Wu, U. S. Patent pending. 